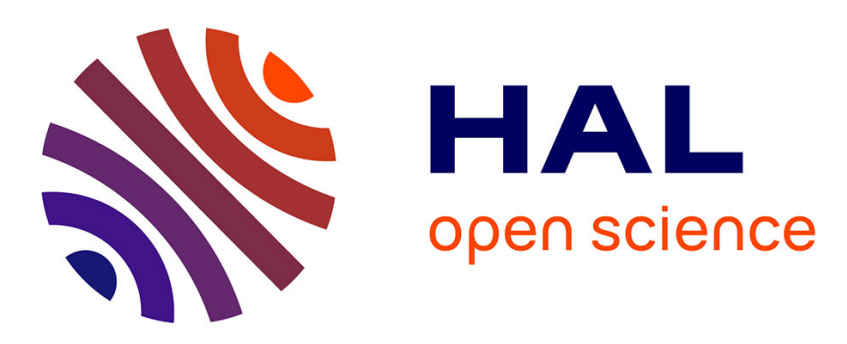

\title{
Real-time dynamic traffic routing using variable structure control
}

Hirsh Majid, Hassane Abouaissa, Daniel Jolly, Gildas Morvan

\section{To cite this version:}

Hirsh Majid, Hassane Abouaissa, Daniel Jolly, Gildas Morvan. Real-time dynamic traffic routing using variable structure control. 2013 16th International IEEE Conference on Intelligent Transportation Systems - (ITSC 2013), Oct 2013, La Haye, Netherlands. pp.1278-1283, 10.1109/ITSC.2013.6728407 . hal-03353679

\section{HAL Id: hal-03353679 https://hal.science/hal-03353679}

Submitted on 10 Oct 2021

HAL is a multi-disciplinary open access archive for the deposit and dissemination of scientific research documents, whether they are published or not. The documents may come from teaching and research institutions in France or abroad, or from public or private research centers.
L'archive ouverte pluridisciplinaire HAL, est destinée au dépôt et à la diffusion de documents scientifiques de niveau recherche, publiés ou non, émanant des établissements d'enseignement et de recherche français ou étrangers, des laboratoires publics ou privés. 


\title{
Real-Time Dynamic Traffic Routing Using Variable Structure Control
}

\author{
Hirsh Majid ${ }^{1}{ }^{2}$, Hassane Abouaïssa ${ }^{1}$, Daniel Jolly ${ }^{1}$, Gildas Morvan ${ }^{1}$
}

\begin{abstract}
In this paper, we present a new algorithm to deal with the real-time dynamic traffic routing (DTR) problem using a second order traffic flow model (METANET). We investigate variable structure control (VSC) as a high-speed switched feedback control resulting in sliding mode. The control objective is to minimize the difference of the travel times on alternate routes in a network setting. Accordingly, the congestion could be reduced/eliminated. The relevance of the proposed algorithm is demonstrated via a set of numerical simulations in the case of the same as well as different geometric conditions.
\end{abstract}

Keywords: Macroscopic model, variable structure control, sliding mode control, dynamic traffic routing.

\section{INTRODUCTION}

Traffic control represents an efficient way to improve the freeway throughput and to ensure an efficient, safe and less polluting transportation of goods and persons [1]. It also contributes to a high reduction of direct and indirect costs.

Freeway traffic control can be achieved via a set of actions and measurements such as: dynamic speed limits, route guidance, dynamic assignment and dynamic traffic routing, ramp metering, etc.

Dynamic traffic assignment (DTA) and dynamic traffic routing (DTR) represent some of the most efficient solutions to steer congestion problems especially the no-recurrent ones. DTR/DTA or control of traffic diversion rests on the determination of time-dependent split variables at the diversion point in order to achieve a user-equilibrium traffic pattern [2] in a way that one route not be overloaded and the others underused. Accordingly, the congestion could be alleviated/eliminated. Several algorithms have been developed to solve this problem. Most of them rely on optimization approaches such as those proposed by [3]. As stated in [4], the proposed optimization algorithm attempts to solve the DTR problem by optimizing the objective functions for the nominal model over the planning horizon and it is not adapted for on-line DTR control. Papageorgiou [5] and Messmer \& Papageorgiou [6] have proposed algorithms for dynamic traffic assignment (DTA) based on the linear quadratic regulator, and nonlinear optimization techniques. Other techniques use expert systems to deal with the diversion problem [7]. Liu \& al. [8] have proposed a strategy based on a model reference adaptive control in order to guide the real-world traffic flow to evolve towards the desired states

1 Univ. Lille Nord France, F-59000 Lille, France. UArtois, LGI2A, EA. 3926 Technoparc Futura, F-62400 Béthune, France. (hassane.abouaissa, daniel.jolly, gildas.morvan) @univ-artois.fr.

2 (Corresponding author). University of Sulaimani, Faculty of Engineering Sciences, Department of Civil Engineering, Sulaimani, Iraq hirshmajid@yahoo.com.

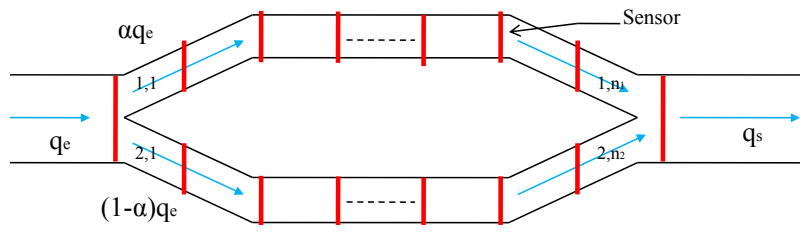

Fig. 1. Sample example of two alternate routes

especially under emergency evacuation. Kachroo \& Özbay [9] have designed feedback and fuzzy control laws for an on-line diversion problem. Another algorithm for DTR and DTA problems based on the nonlinear $H_{\infty}$ was proposed in [10]. See also [11] and [12] for more information about route guidance strategy.

In this paper, we exploit the variable structure control theory (VSC). The control algorithm consists of two parts; trajectory planning (open loop control) which is achieved using a specific class of complex systems called 'Differentially flat systems'. The trajectory tracking (closed loop control) is ensured by a high-speed switched feedback control resulting in sliding mode. In contrast of [13], the non-destination oriented METANET model [14] [15] is used in the design of the control algorithm.

The paper is organized as follows: Section II presents the main principles of the DTR problem and its mathematical formulation. Section III recalls the main definitions of the control system. Section IV shows the control design methodology using the concept of flatness and sliding mode control. Section V provides some numerical simulations for a sample network. Section VI concludes the paper and outlines some tracks for further developments.

\section{DYNAMIC TRAFFIC ROUTING FORMULATION}

In this section, we first present a mathematical formulation which is used for the design of DTA/DTR flat controller. We expose a second order traffic flow model ( METANET).

For the sake of simplicity, we consider the case of two alternate routes divided into $n_{1}$ and $n_{2}$ sections, respectively, as depicted in Fig. 1.

The proposed control approach rests on the use of METANET model. The dynamic equation of the traffic density reads:

$$
\dot{\rho}_{i, j}(t)=\frac{1}{L_{i, j}}\left[q_{i, j-1}(t)-q_{i, j}(t)\right]
$$

where $\quad(i, j)=(1,1),(1,2), \ldots,\left(1, n_{1}\right),(2,1), \ldots,\left(2, n_{2}\right)$. $L_{i, j}$ is the section length and $\rho_{i, j}$ represents the traffic 
density at section $j$ of the route $i$.

The relation between the traffic flow $q_{i, j}$ and the mean speed $v_{i, j}$ is:

$$
q_{i, j}(t)=\rho_{i, j}(t) v_{i, j}(t) \lambda_{i, j}
$$

where $\lambda_{i, j}$ is the number of lanes. $v_{i, j}$ is the dynamic mean speed of section $j$ on the route $i$.

The equation of dynamic mean speed is:

$$
\begin{aligned}
\dot{v}_{i, j}(t)= & \frac{1}{\tau}\left(V_{\rho_{i, j}}(t)-v_{i, j}(t)\right)+\frac{1}{L_{i, j}} v_{i, j}(t)\left(v_{i, j-1}(t)\right. \\
& \left.-v_{i, j}(t)\right)-\frac{\nu}{\tau L_{i, j}} \frac{\rho_{i, j+1}(t)-\rho_{i, j}(t)}{\rho_{i, j}(t)+\kappa}
\end{aligned}
$$

$V_{\rho_{i, j}}$ is defined as a nonlinear expression called fundamental diagram mean speed (see [16]):

$$
V_{\rho_{i, j}}(t)=v f_{i, j} \exp \left[\left(-\frac{1}{a}\right)\left(\frac{\rho_{i, j}(t)}{\rho_{c_{i, j}}}\right)^{a}\right]
$$

$\rho_{c_{i, j}}, v f_{i, j}, a, \nu, \kappa$ and $\tau$ are constant parameters which reflect particular characteristics of a given traffic system [14].

The control input $\alpha(t) \in[0,1]$ is defined as an exogenous variable of the system [12], it is a split rate that allows to reach a user equilibrium traffic pattern. For the case of two alternate routes, the equations of entry flow in each route are:

$$
\left\{\begin{array}{cc}
\alpha(t) q_{e}(t)= & q_{1, i n}(t) \\
(1-\alpha) q_{e}(t)= & q_{2, \text { in }}(t)
\end{array}\right.
$$

where $q_{e}(t)$ is the traffic demand. The control objective is to find the optimal split rate $\alpha$ in order to minimize the differences $J(\alpha)$ between the travel time $T T$ of the two alternate routes.

Kachroo and Özbay [4] have formulated the DTR problem as follows: finding $\alpha_{0}$ and the optimal $\alpha(t)$, which minimizes

$$
J(\alpha)=\int_{0}^{t_{f}}\left[\sum_{j=1}^{n_{1}} T T\left(\rho_{1, j}\right)-\sum_{j=1}^{n_{2}} T T\left(\rho_{2, j}\right)\right]^{2} d t
$$

where $T T\left(\rho_{1, j}\right)$ and $T T\left(\rho_{2, j}\right)$ are the travel times function of section $j$ on the route 1 and 2 respectively. $t_{f}$ is the final time.

In the general case, consider a traffic flow system with $n$ alternate routes. The system is described by the same ordinary differential equation (1) where, $(i, j)=$ $\left((1,1), \ldots,\left(1, n_{1}\right),(2,1), \ldots,\left(2, n_{2}\right),(n, 1), \ldots,\left(n, n_{n}\right)\right)$.

The problem consists then in finding a set of split variables, $\alpha_{n-1}$, where, $\sum_{i=1}^{n-1} \alpha^{i}=1$, that minimizes total travel time $T T$, (see [4], for optimal formulation of the DTR problem for a general case)

$$
\left\{\begin{array}{cl}
\alpha_{1}(t) q_{e}(t)= & q_{1, i n}(t) \\
\alpha_{2}(t) q_{e}(t)= & q_{2, \text { in }}(t) \\
\vdots & \\
\left(1-\alpha_{1}-\alpha_{2}-, \ldots,-\alpha_{n-1}\right) q_{e}(t)= & q_{n, i n}(t)
\end{array}\right.
$$

Note that for such a complex problem, an open loop control structure is not sufficient [10], it calls for a more robust feedback control one. In this context, we highlight the interest of an attractive approach based on the concept of differential flatness [17] [18]. The following section, recalls the main definitions and principles of differentially flat systems.

\section{SLIDING MOdE FLATNESS-BASED CONTROL SYSTEMS}

In this paper, we exploited one of the most attractive methods that can be applied to a broad class of nonlinear systems resulting in controllers that are robust to modeling errors and unknown disturbances. We investigated variable structure control (VSC) as a high-speed switched feedback control resulting in sliding mode. The gains in each feedback path switch between two values according to a rule that depends on the value of the state at each instant. The purpose of the switching control law is to derive the non-linear system's state trajectory onto a prespecified surface in the state space and to maintain the system's state trajectory on this surface (switching surface) for subsequent time.

In standard sliding mode control, or first order sliding mode control (FOSMC) [19] and [20], the sliding surface is chosen so that it has a relative degree of one with respect to the control input. In such a case, the control input $u$ is, for example, of the form;

$u(t)=u_{e q}(t)+u_{d}(t)$, where $u_{e q}$ is the continuous function and $u_{d}(t)=-K_{d} \operatorname{sign}(s(t))-K_{p} s(t)$ is the discontinuous function. $K_{d}$ specifies the speed of convergence of the closed-loop system in order to $s(t)=0$. To combine a small switching gain with fast convergence, the discontinuous control term could be extended with a proportional feedback term $K_{p} s(t)$ [21].

$u_{e q}$ ensures that $\dot{s}=0$. It is the equivalent control, it depends only on the switching surface $s(t)$ and not on the control function $u_{d}$ [20]. Thus, it can be said that $u_{e q}$ introduces the trajectory planning (open loop control) of the output. In this context, the term $u_{d}$ should introduce the trajectory tracking (closed loop control) of the output.

In this paper, instead of $u_{e q}$, the trajectory is made using a specific class of control system called 'differential flatness'

The concept of flat systems was first introduced by Fliess and al [17] [18] more than a decade ago using the formalism of differential algebra (see [22] for a slightly different approach of differentially flat systems). This special class of non-linear control systems described by ordinary differential equations: differentially flat systems form a special class of nonlinear control systems for which systematic control methods are available once a flat-output is explicitly known.

The flatness-based control methods may be expected to play a very significant role in high technology applications in the next few years, similar to what happened for nonlinear control in the last decade [23]. The main property of flat systems is that all the state and input variables can be expressed directly, without integration of any differential equation, in terms of the set of so-called "flat output" and a number of its time derivatives. More precisely, the entire system behavior is determined by the trajectory of a finite collection of quantities: flat outputs. This leads to a simple and elegant trajectory design. For a given system, the number 


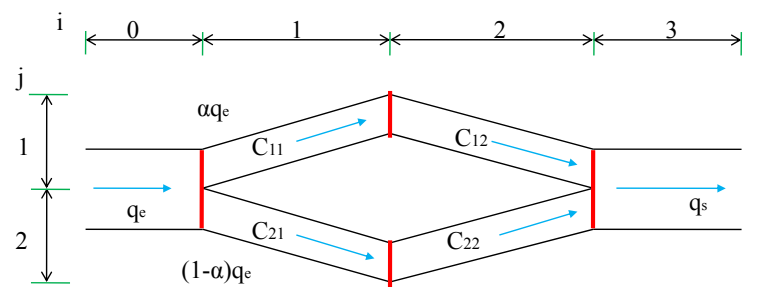

Fig. 2. Simulated freeway section

of flat outputs is equal to the number of the system inputs. For more details on flatness see also [18], [23], [24], [25], [26] and [27].

\section{SLIDING MODE FLATNESS-BASED CONTROL FOR THE DTR PROBLEM}

Here, we introduce a first order sliding mode flatnessbased control using the METANET model for dynamic traffic routing. In the freeway portion, there are two alternate routes with two sections in each route, see Fig. 2.

A. DTR Control for Two Alternate Routes With Two Sections The equations of the system discretized in space are

$$
\begin{gathered}
\dot{\rho}_{11}(t)=\frac{1}{L_{11}}\left[\alpha q_{e}-\rho_{11}(t) v_{11}(t)\right] \\
\dot{v}_{j i}(t)=\frac{1}{\tau}\left(V_{\rho_{j i}}(t)-v_{j i}(t)\right)+\frac{1}{L_{j i}} v_{j i}(t)\left(v_{j i-1}(t)\right. \\
\left.-v_{j i}(t)\right)-\frac{\nu}{\tau L_{j i}} \frac{\rho_{j i+1}(t)-\rho_{j i}(t)}{\rho_{j i}(t)+\kappa} \\
\dot{\rho}_{21}(t)=\frac{1}{L_{21}}\left[(1-\alpha) q_{e}-\rho_{21}(t) v_{21}(t)\right] \\
\dot{\rho}_{j 2}(t)=\frac{1}{L_{j 2}}\left[\rho_{j 1}(t) v_{j 1}(t)-\rho_{j 2}(t) v_{j 2}(t)\right] \\
V_{\rho_{j i}}(t)=v f_{j i} \exp \left[\left(-\frac{1}{a}\right)\left(\frac{\rho_{j i}(t)}{\rho_{c_{j i}}}\right)^{a}\right]
\end{gathered}
$$

The travel time function is obtained as follows:

$$
\begin{aligned}
& y_{1}(t)=\frac{L_{11}}{v_{11}(t)}+\frac{L_{12}}{v_{12}(t)} \\
& y_{2}(t)=\frac{L_{21}}{v_{21}(t)}+\frac{L_{22}}{v_{22}(t)}
\end{aligned}
$$

The variable $y$ is equal to the difference in the travel times the two sections.

$$
y(t)=\left[\left(\frac{L_{11}}{v_{11}(t)}+\frac{L_{12}}{v_{12}(t)}\right)-\left(\frac{L_{21}}{v_{21}(t)}+\frac{L_{22}}{v_{22}(t)}\right)\right]
$$

The first time derivative leads to:

$$
\dot{y}(t)=A+B+C+D+\alpha E
$$

The terms A, B, C, and D represent derivatives of sections 21, 11, 12 and 22 respectively. The term E consists of all the terms in which $\alpha$ does exist. It comes from sections 11 and 21. $\mathrm{A}$ is composed of three terms $\left(A_{1}, A_{2}\right.$ and $\left.A_{3}\right)$;

$$
\begin{gathered}
A_{1}=\frac{L_{21}}{\left(v_{21}\right)^{2}}\left[\frac{\frac{v_{21}-\frac{v f_{21}}{\sigma_{4}}}{\tau}-\frac{\nu \sigma_{6}}{L_{21} \tau\left(\kappa+\rho_{21}\right)}}{\tau}-\frac{\frac{\nu\left(\rho_{21} v_{21}-\rho_{22} v_{22}\right)}{L_{22}}}{L_{21} \tau\left(\kappa+\rho_{21}\right)}\right] \\
A_{2}=\frac{L_{21}}{v_{21}} \rho_{21}\left[\frac{\frac{v f_{21} \sigma_{8}}{L_{21} \rho_{21} \sigma_{4}}}{\tau}-\frac{\frac{\nu}{L_{21}}}{L_{21} \tau\left(\kappa+\rho_{21}\right)}+\frac{\nu \sigma_{6}}{\left(L_{21}\right)^{2} \tau \sigma_{9}}\right] \\
A_{3}=\frac{L_{21} q_{e}}{\left(v_{21}\right)^{2}}\left[\frac{-\frac{v f_{21} \sigma_{8}}{L_{21} \rho_{21} \sigma_{4}}}{\tau}+\frac{\frac{\nu}{L_{21}}}{L_{21} \tau\left(\kappa+\rho_{21}\right)}-\frac{\nu \sigma_{6}}{\left(L_{21}\right)^{2} \tau \sigma_{9}}\right]
\end{gathered}
$$

B is composed of two terms $\left(B_{1}\right.$ and $\left.B_{2}\right)$;

$$
\begin{gathered}
B_{1}=\frac{-L_{11}}{\left(v_{11}\right)^{2}}\left[\frac{\frac{v_{11}-\frac{v f_{11}}{\sigma 3}}{\tau}-\frac{\nu \sigma_{5}}{L_{11} \tau\left(\kappa+\rho_{11}\right)}}{\tau}-\frac{\frac{\nu\left(\rho_{11} v_{11}-\rho_{12} v_{12}\right)}{L_{12}}}{L_{11} \tau\left(\kappa+\rho_{11}\right)}\right] \\
B_{2}=\frac{-L_{11}}{v_{11}} \rho_{11}\left[\frac{\frac{v f_{11} \sigma_{7}}{L_{11} \rho_{11} \sigma_{3}}}{\tau}-\frac{\frac{\nu}{L}}{L_{11} \tau\left(\kappa+\rho_{11}\right)}+\frac{\nu \sigma_{5}}{\left(L_{11}\right)^{2} \tau \sigma_{10}}\right]
\end{gathered}
$$

$\mathrm{C}$ is composed of three terms $\left(C_{1}, C_{2}\right.$ and $\left.C_{3}\right)$;

$$
\begin{gathered}
C_{1}=\frac{-L_{12}}{\left(v_{12}\right)^{2}}\left[\frac{\left.-\sigma_{13}+\frac{v_{12}-\frac{v f_{12}}{\sigma_{11}}}{\tau}-\frac{v f_{12} \sigma_{15}\left(\rho_{11} v_{11}-\rho_{12} v_{12}\right)}{L_{12} \rho_{12} \sigma_{11}}\right]}{\tau}\right] \\
C_{2}=\frac{1}{v_{12}}\left[\frac{v_{11}-\frac{v f_{11}}{\sigma_{19}}}{\tau}-\frac{v_{12}-\frac{v f_{12}}{\sigma_{11}}}{\tau}+\sigma_{13}-\frac{\nu\left(\rho_{11}-\rho_{12}\right)}{L_{11} \tau\left(\kappa+\rho_{11}\right)}\right] \\
C_{3}=\frac{\sigma_{17}}{\left(v_{12}\right)^{2}}\left[\frac{v_{12}-\frac{v f_{12}}{\sigma_{11}}}{\tau}-\sigma_{13}\right]
\end{gathered}
$$

$\mathrm{D}$ is composed of three terms $\left(D_{1}, D_{2}\right.$ and $\left.D_{3}\right)$;

$$
\begin{gathered}
D_{1}=\frac{L_{22}}{\left(v_{22}\right)^{2}}\left[\frac{\left.-\sigma_{14}+\frac{v_{22}-\frac{v f_{22}}{\sigma_{12}}}{\tau}-\frac{v f_{22} \sigma_{16}\left(\rho_{21} v_{21}-\rho_{22} v_{22}\right)}{L_{22} \rho_{22} \sigma_{12}}\right]}{\tau}\right] \\
D_{2}=\frac{-1}{v_{22}}\left[\frac{v_{21}-\frac{v f_{21}}{\tau} \sigma_{20}}{\tau}-\frac{v_{22}-\frac{v f_{22}}{\sigma_{12}}}{\tau}+\sigma_{14}-\frac{\nu\left(\rho_{21}-\rho_{22}\right)}{L_{21} \tau\left(\kappa+\rho_{21}\right)}\right] \\
D_{3}=-\frac{\sigma_{18}}{\left(v_{22}\right)^{2}}\left[\frac{v_{22}-\frac{v f_{22}}{\sigma_{12}}}{\tau}-\sigma_{14}\right]
\end{gathered}
$$

$\mathrm{E}$ is composed of two terms. The first one concerns section 11 and the second belongs to section 21 ;

$$
\begin{aligned}
& E_{11}=\frac{L_{11} q_{e}}{\left(v_{11}\right)^{2}}\left[\frac{\frac{v f_{11} \sigma_{7}}{L_{11} \rho_{11} \sigma_{3}}}{\tau}-\frac{\frac{\nu}{L_{11}}}{L_{11} \tau\left(\kappa+\rho_{11}\right)}+\frac{\nu \sigma_{5}}{\left(L_{11}\right)^{2} \tau \sigma_{10}}\right] \\
& E_{21}=\frac{L_{21} q_{e}}{\left(v_{21}\right)^{2}}\left[\frac{\frac{v f_{21} \sigma_{8}}{L_{21} \rho_{21} \sigma_{4}}}{\tau}-\frac{\frac{\nu}{L_{21}}}{L_{21} \tau\left(\kappa+\rho_{21}\right)}+\frac{\nu \sigma_{6}}{\left(L_{21}\right)^{2} \tau \sigma_{9}}\right]
\end{aligned}
$$

$\sigma_{3}=\exp \left(\frac{1}{a}\left(\frac{\rho_{11}}{\rho_{c_{11}}}\right)^{a}\right), \quad \sigma_{4}=\exp \left(\frac{1}{a}\left(\frac{\rho_{21}}{\rho_{c_{21}}}\right)^{a}\right)$, $\sigma_{5}=\rho_{11}-\rho_{12}, \sigma_{6}=\rho_{21}-\rho_{22}, \sigma_{7}=\left(\frac{\rho_{11}}{\rho_{c_{11}}}\right)^{(a-1)}$, $\sigma_{8}=\left(\frac{\rho_{21}}{\rho_{c_{21}}}\right)^{(a-1)}, \sigma_{9}=\left(\kappa+\rho_{21}\right)^{2}, \sigma_{10}=\left(\kappa+\rho_{11}\right)^{2}$, $\sigma_{11}=\exp \left(\frac{1}{a}\left(\frac{\rho_{12}}{\rho_{c_{12}}}\right)^{a}\right), \quad \sigma_{12}=\exp \left(\frac{1}{a}\left(\frac{\rho_{22}}{\rho_{c_{22}}}\right)^{a}\right)$, $\sigma_{13}=\frac{v_{12} \sigma_{17}}{L_{12}}, \sigma_{14}=\frac{v_{22} \sigma_{18}}{L_{22}}, \sigma_{15}=\left(\frac{\rho_{12}}{\rho_{c_{12}}}\right)^{(a-1)}$, $\sigma_{16}=\left(\frac{\rho_{22}}{\rho_{c_{22}}}\right)^{(a-1)}, \sigma_{17}=v_{11}-v_{12}, \sigma_{18}=v_{21}-v_{22}$, $\sigma_{19}=\exp \left(\frac{1}{a}\left(\frac{\rho_{11}}{\rho_{c_{11}}}\right)^{a}\right)$ and $\sigma_{20}=\exp \left(\frac{1}{a}\left(\frac{\rho_{21}}{\rho_{c_{21}}}\right)^{a}\right)$. 
The studied system is characterized by one input (control) variable, we have then one flat output $F=y$, which represents the difference in travel time function between the two routes. Thus the equation (15) can be rewritten as:

$$
\dot{F}(t)=A+B+C+D+\alpha E
$$

from which

$$
\alpha(t)=\frac{\dot{F}(t)-(A+B+C+D)}{E}
$$

The expression of the state variable allows to choose a suitable trajectory of the travel time (the flat output). The equation of the control (input) variable allows to add additional constraints to this travel time trajectory. This means that all important properties of the system (see equation (15)) are contained in such a differential parametrization.

\section{B. Trajectory Planning:}

The equation (17), corresponds to an open loop control algorithm. In order to define the trajectory planning, a suitable desired trajectory $F^{*}$ has to be defined. According to the expression of the control variable in equation (17), this trajectory must have smooth derivatives up to order two. In order to reduce computational effort in real time situation, one can build this reference trajectory for the travel time (flat output) using a polynomial interpolation [23] from the initial and final conditions of the travel time $\left(F\left(t_{i}\right)=F_{i}, \dot{F}\left(t_{i}\right)=0\right)$ and $\left.\left(F\left(t_{f}\right)=F_{f}, \dot{F}\left(t_{f}\right)\right)=0\right)$. This is accomplished by prescribing the following desired trajectory for the flat output $F$ :

$F^{*}(t)= \begin{cases}F_{t_{i}} & \text { for } t<t_{i} \\ F_{t_{i}}+\left(F_{t_{f}}-F_{t_{i}}\right) \varphi\left(t, t_{i}, t_{f}\right) & \text { for } t_{i} \leq t \leq t_{f} \\ F_{t_{f}} & \text { for } t>t_{f}\end{cases}$

where $\varphi\left(t, t_{i}, t_{f}\right)$ is a polynomial function of time which exhibit a sufficient number of zero derivatives at times $t_{i}$ and $t_{f}$. (For the polynomial calculation see e.g. [28] [23]):

$$
\varphi(t)= \begin{cases}0 & \text { if } t<t_{i} \\ 3\left(\frac{t-t_{i}}{t_{f}-t_{i}}\right)^{2}-2\left(\frac{t-t_{i}}{t_{f}-t_{i}}\right)^{3} & \text { if } t_{i} \leq t \leq t_{f} \\ 1 & \text { if } t>t_{f}\end{cases}
$$

Thus, by replacing the term $\dot{F}(t)$ in equation (17) by the term of desired trajectory $\dot{F}^{*}(t)$, we obtain the nominal open loop control:

$$
\alpha^{*}(t)=\frac{\dot{F}^{*}(t)-(A+B+C+D)}{E}
$$

\section{Trajectory Tracking:}

Due to the parameter variations and disturbances in traffic flow and frequent changes in the traffic conditions, the open loop control is not sufficient. To ensure a steady state and reduce the influence of parameter variations, the traffic flow has to be operated in closed loop. Using the flatness-based open loop presented in equation (17), an additional feedback can be determined in order to achieve a desired dynamic behaviour and to compensate the external

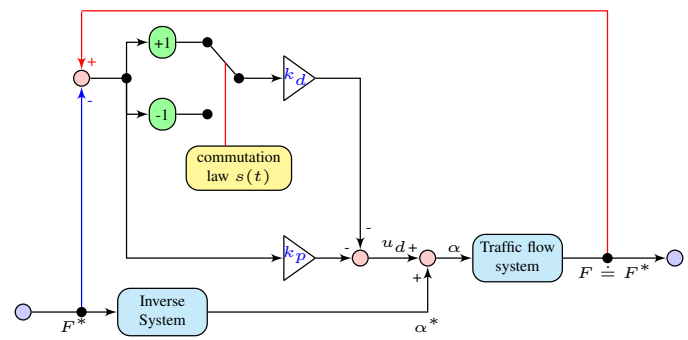

Fig. 3. Closed loop control structure

TABLE I

THE MODEL AND SIMULATION PARAMETERS

\begin{tabular}{cccc}
\hline \hline Parameter & value & Parameter & value \\
\hline & & & \\
a & 2.34 & $\rho_{c}$ & $36 \mathrm{veh} / \mathrm{km}$. \\
$\tau$ & $18 \mathrm{~s}$ & $\rho_{\max }$ & $180 \mathrm{veh} / \mathrm{km}$. \\
$\nu$ & $60 \mathrm{~km}^{2} / \mathrm{h}$ & $v f$ & $90 \mathrm{~km} / \mathrm{h}$. \\
$\kappa$ & $40 \mathrm{veh} / \mathrm{km}$ & $q_{\max }$ & $\rho_{c} v f \exp \left(\frac{-1}{\mathrm{a}}\right)$. \\
Time step & $20 \mathrm{~s}$ & Cell length & $400 \mathrm{~m}$ \\
\hline
\end{tabular}

disturbances. Here, a first order sliding mode controller is used to feedback the system which asymptotically regulates the output towards the desired equilibrium position (see Fig. 3). Thus, the closed-loop control schema including the flatness-based loop can be obtained as follows:

$$
\psi(t)=\dot{F}^{*}(t)-K_{d} \operatorname{sign}(s(t))-K_{p} s(t)
$$

where $k_{d}$ and $k_{p}$ are parameters that must be selected so as to satisfy the desired performances of the closed loop system and to ensure asymptotically stabilization of the input variable. The control law $\alpha$ now reads:

$$
\begin{aligned}
\alpha(t)= & {\left[\frac{\dot{F}^{*}(t)-K_{d} \operatorname{sign}(s(t))-K_{p} s(t)}{E}\right.} \\
& \left.-\frac{A+B+C+D}{E}\right]
\end{aligned}
$$

\section{NUMERICAL SIMULATIONS}

For the numerical simulations, consider the freeway section depicted in Fig. 2. It shows two routes with the same length and geometric conditions. Each route is partitioned into 2 identical cells (each cell $=400 \mathrm{~m}$ ). At the end of each cell, one loop detector (sensor) is installed. The model parameters are depicted in table I. The control algorithm value is shown via variable message signs (VMS). For the sake of simplicity, we assume a full compliance of the drivers.

The used data is collected between $6 \mathrm{AM}-10 \mathrm{PM}$. The simulation time step is about $20 \mathrm{~s}$. The simulations have been done in two different cases; when the two routes have the same geometric conditions as well as when the two routes have different geometric conditions $(\mathrm{L} 11, \mathrm{~L} 12=300 \mathrm{~m})$. The traffic demand used in the simulations is shown in Fig. 4. Fig. 5, 6, 7 and 8 show the densities, mean speeds, control values and the travel times evolution in the case when the two routes have the same geometric conditions. From these 


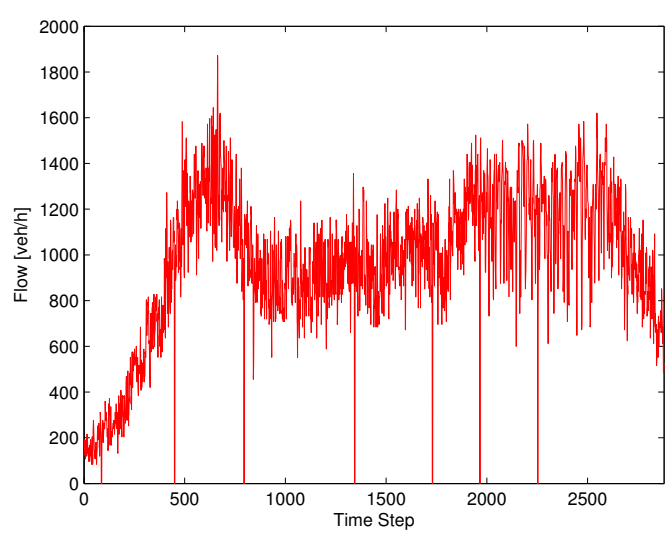

Fig. 4. Traffic demand used in the simulation
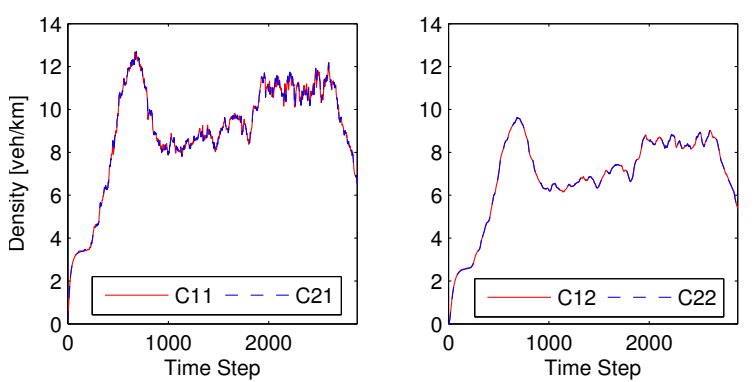

Fig. 5. Traffic densities in the case of the same geometric conditions

figures, it is clear that the densities and mean speeds of the system in each reciprocal cell are equal, it means that the control algorithm distribute the traffic demand between two routes somehow one route not be overloaded and the other underused. Fig. 9, 10, 11 and 12 show the densities, mean speeds, control values and the travel times evolution in the case when the two routes have some different in theirs geometric conditions $(\mathrm{L} 11, \mathrm{~L} 12=300 \mathrm{~m}, \mathrm{~L} 21, \mathrm{~L} 22=400$ $\mathrm{m})$. We can realize the control algorithm distribute always the traffic demand between the two routes equally. Contrary, because of different length of the two routes, the mean speed of the two routes are different.
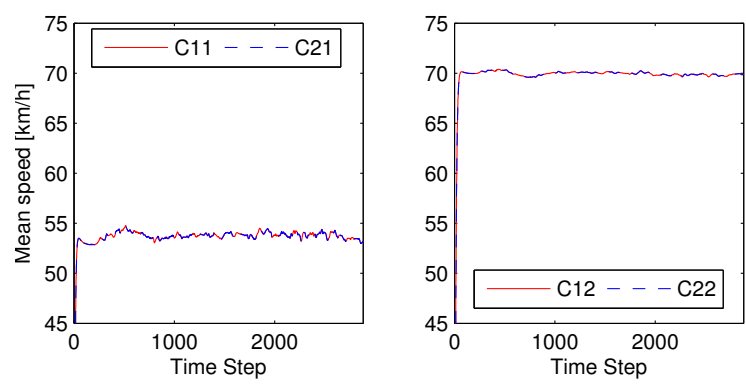

Fig. 6. Mean speeds in the case of the same geometric conditions
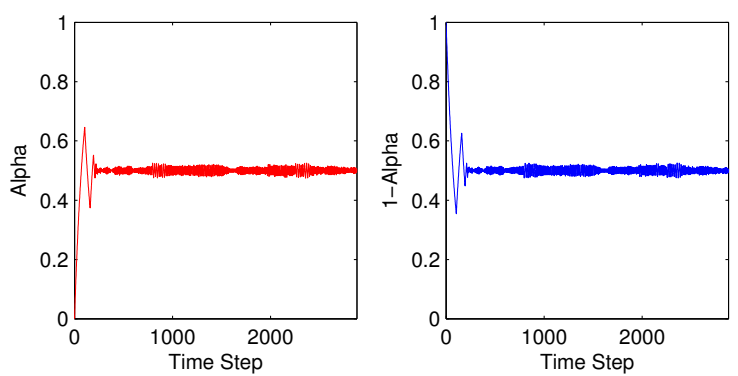

Fig. 7. Control value in the case of the same geometric conditions
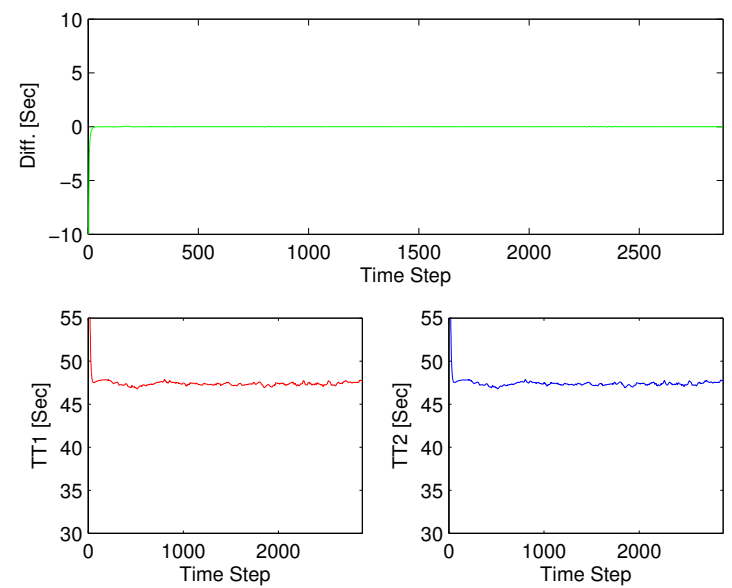

Fig. 8. Travel times of the two routes with the same geometric conditions
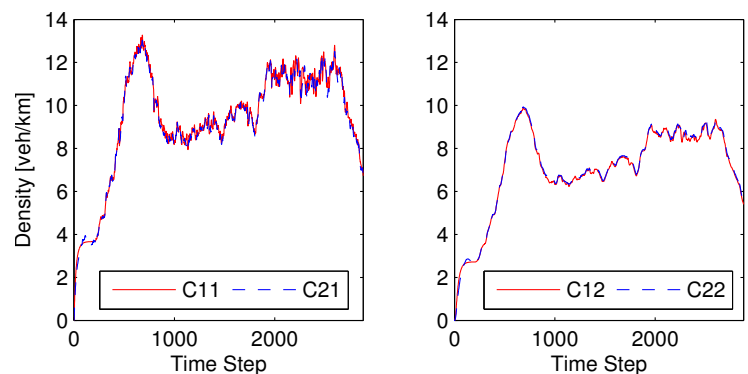

Fig. 9. Traffic densities in the case of different geometric conditions
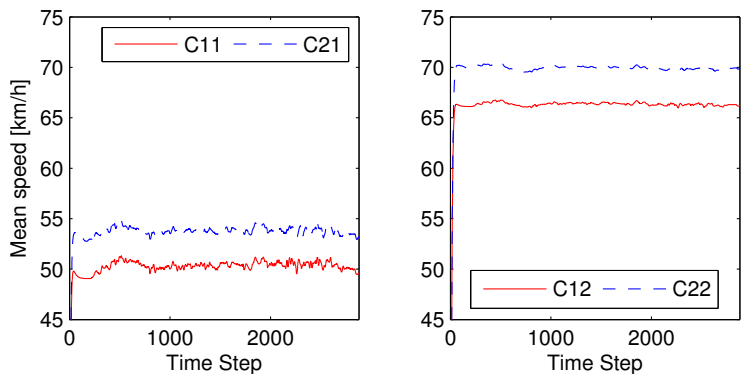

Fig. 10. Mean speeds in the case of different geometric conditions 

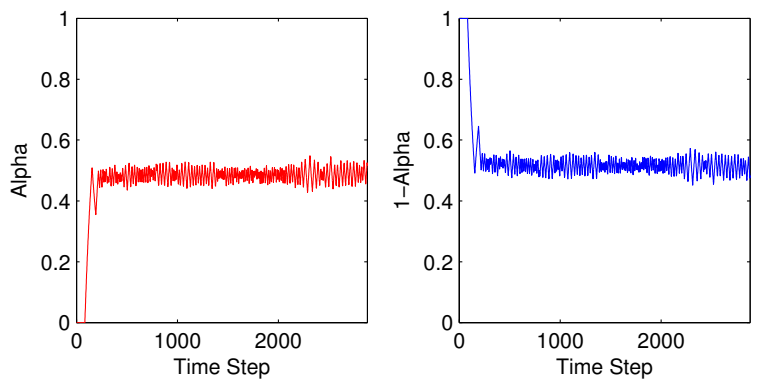

Fig. 11. Control value in the case of different geometric conditions
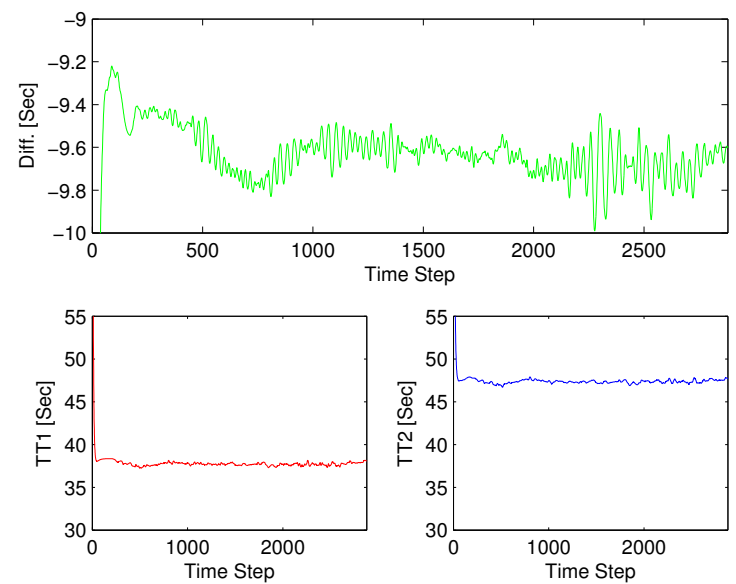

Fig. 12. Travel times of the two routes with different geometric conditions

\section{CONCLUSION}

In this paper, a first order sliding mode flatness-based control algorithm was designed in order to control the incoming flow into two alternate routes which have the same origin and destination. The objective of the control algorithm is to minimize the difference between the travel times of two alternate routes, accordingly, to optimize the flow on the freeway portion. The control algorithm was applied using the METANET model which is a nonlinear traffic flow model. The results show the relevance of the control algorithm in the cases of the same and different geometric conditions of the two alternate routes. These results inspire us to extend this work to deal with more complex network and to design an integrated control in the future.

\section{REFERENCES}

[1] A. Kostialos and M. Papageorgiou. Motorway network traffic control systems. European Journal of Operational Research, vol. 152, pages:321-333, 2004.

[2] P. Kachroo and K. Özbay. Solution to the user equilibrium dynamic traffic routing problem using feedback linearization. Transp. Res. B, vol. No. 5, pages:343-360, 1998.

[3] S. Petta and H. Mahmassani. Multiple users classes real time traffic assignement for on-line operations: a rolling horizon solution framework. Trans. Res. C, vol. No. 3, pages:83-98, 1995.
[4] P. Kachroo and K. Özbay. System dynamics and feedback control problem formulations for real-time dynamic traffic routing. Math. Comput. Modelling, Vol.27 N 9-11, pages:27-49, 1998.

[5] M. Papageorgiou. Dynamic modeling, assignment and route guidance traffic networks. Trans. Res. B, vol. No. 24, pages:471-495, 1990.

[6] A. Messmer and M. Papageorgiou. Route diversion control in motorway networks via nonlinear optimization. IEEE Trans. on Control Systems Tech. 3(1), 1995.

[7] A. Gupta, V. Maslanka, and G. Spring. Development of prototype kbes in the management of non-recurrent congestion of the massachusetts turnpike. 17st Annual Meeting of TRB, Washington, DC., 1992.

[8] H. X. Liu, J.X. Ban, W. Ma, and P. B. Michandani. Model reference adaptive control framework for real-time traffic management under emergency evacuation. Journal of urban Planning and Development, ASCE, pages :43-50, 2007.

[9] P. Kachroo and K. Özbay. Real-time dynamic traffic routing based on fuzzy feedback control methodology. Transportation Research Record, vol. N. 1556, 1996.

[10] P. Kachroo and K. Özbay. Feedback control solutions to network level user-equilibrium real-time dynamic traffic assignement problems. Networks and spacial Economics, vol. 5:pages: 243-260, 2005.

[11] Abdes Karimi, Andreas Hegyi, Bart De Schutter, Hans Hellendoorn, and Frans Middelham. Integration of dynamic route guidance and freeway ramp metering using model predictive control. In Proceeding of the 2004 American Control Conference. Boston, Massachusetts, June 30 - July 2, 2004.

[12] Y. Pavlis and M. Papageorgiu. Simple decentralized feedback strategies for route guidance in traffic networks. Transportation science, vol.33, pages:264-278, 1999.

[13] H. Abouaiissa, D. Jolly, and T. Stoilov. Flatness based control for real-time dynamic traffic routing problem. In 14th IFAC Symposium on Information Control Problems in Manufacturing - IFAC-INCOM'2012, may, 23-25 Bucarest, Romania, 2012.

[14] Technical university of Crete Dynamic Systems, Simulation laboratory, and A. Messemer. Metanet - a simulation program for motorway networks. July, 2000.

[15] A.Messemer and M. Papageorgiou. Metanet: a macroscopic simulation program for motorway networks. Traffic Engineering and Control, vol. 31(9):pages: 446-470, 1990.

[16] A. D. May. Traffic Flow Fundamentals. Prentice Hall, Englewood Cliffs, NJ, 1990.

[17] M. Fliess, J. Lévine, P. Martin, and P. Rouchon. Sur les systèmes non linéaires différentiellements plats. C.R. Acad. Sci., vol. I-315, pages:619-624, 1992.

[18] M. Fliess, J. Lévine, P. Martin, and P.Rouchon. Flatness and defect of non-linear systems: Introductory theory and examples. Int. J. Control, vol. 61, pages:1327-1361, 1995.

[19] Neil Munro PH.D. D.SC., editor. Sliding Mode Control in Engineering. Marcel Dekker, INC., 2002.

[20] Rashid R. Kohan. Robust State Estimation and Control of Highway Traffic Systems. PhD thesis, Electrical and Computer Engineering/ University of Toronto, 2001.

[21] Govert Monsees. Discrete-Time Sliding Mode Control. PhD thesis, University of Delft, 2002.

[22] Ph. Martin. Contribution l'étude des systèmes Différentiellement plat. $\mathrm{PhD}$ thesis, Ecole des Mines de Paris, 1992.

[23] H. Sira-Ramírez and S.K. Agrawal. Differentially flat systems. Marcel Dekker, 2004.

[24] V. Manish. Higher order sliding mode control of differentially flat systems. Master's thesis, Indian Institute of Technology, Bombay, Mumbai, 2004.

[25] A. Isidori, C.H. Moog, and A De Luca. A sufficient condition for full linearization via dynamic state feedback. In In proceedings 25 th. IEEE conf. Decision Control, pages 203-208, 1986.

[26] P. Rouchon and Z. Zamm. Flatness based control of oscillators. Angew Math. Mech, vol. 85(6), pages:411-421, 2005.

[27] Ph. Martin, R. M. Murray, and P. Rouchon. Flat systems. In Minicourse ECC'97 Brussels. 1997.

[28] J. Rudolph. Flatness based control of distributed parameter systems. Shaker Verlag, 2003. 